\title{
Urgences
}

\section{André Gervais, La nuit se lève, Saint-Lambert, Éditions du Noroît, 1990, 173 p. (avec un tableau de Bruno Santerre).}

\section{Jean-Marc Bélanger}

Numéro 29, octobre 1990

\section{Éclats d'œuvre}

URI : https://id.erudit.org/iderudit/025614ar

DOI : https://doi.org/10.7202/025614ar

Aller au sommaire du numéro

\section{Éditeur(s)}

Urgences

\section{ISSN}

0226-9554 (imprimé)

1927-3924 (numérique)

Découvrir la revue

Citer ce compte rendu

Bélanger, J.-M. (1990). Compte rendu de [André Gervais, La nuit se lève, Saint-Lambert, Éditions du Noroît, 1990, 173 p. (avec un tableau de Bruno Santerre).] Urgences, (29), 94-96. https://doi.org/10.7202/025614ar d'utilisation que vous pouvez consulter en ligne.

https://apropos.erudit.org/fr/usagers/politique-dutilisation/ 


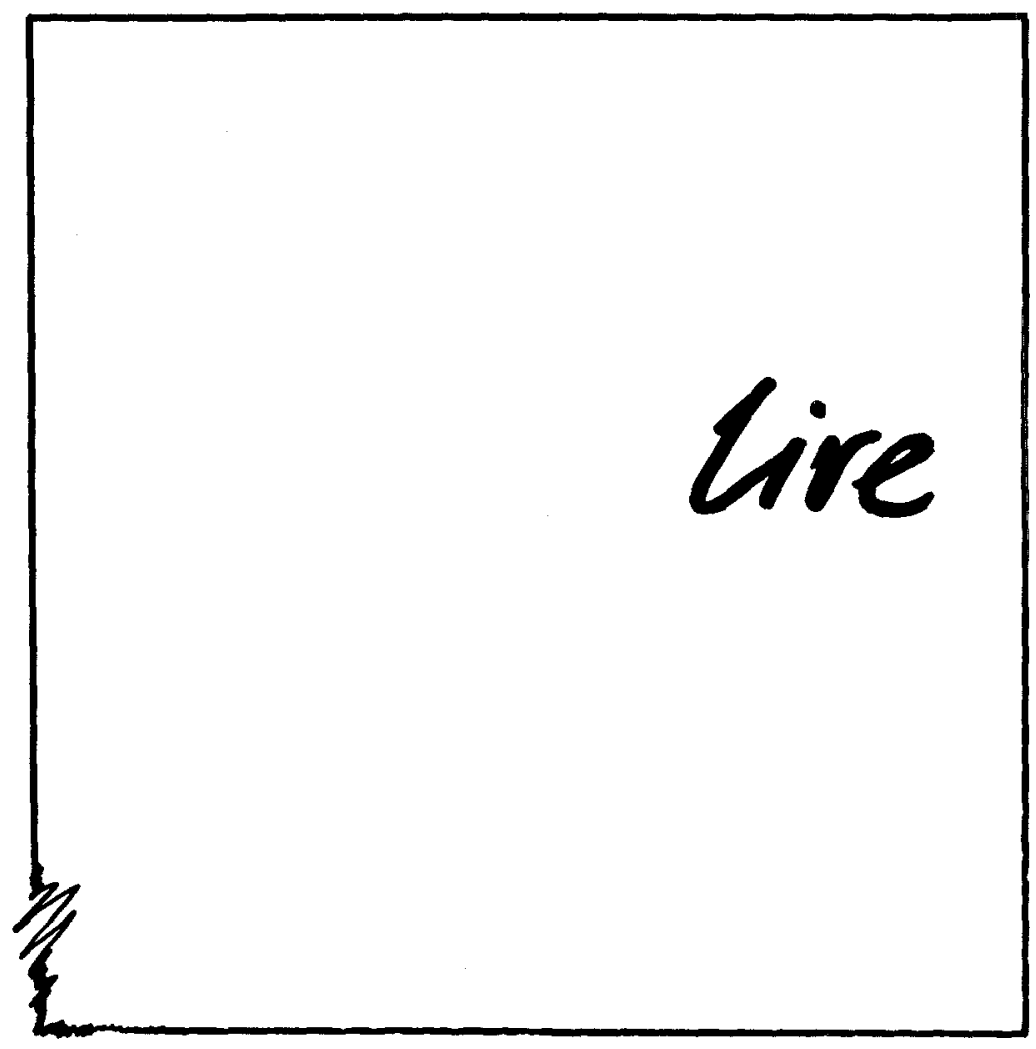




\section{André Gervais, La nuit se lève, Saint-Lambert, Éditions du Noroît, 1990, 173 p. (avec un tableau de Bruno Santerre).}

Le recueil, comme il est précisé en quatrième de couverture, "dispose des textes écrits durant les dix dernières années", certains inédits, d'autres déjà publiés, parfois dans des versions sensiblement différentes. Malgré les écarts temporels qui séparent ces textes les uns des autres et leur apparente hétérogénéité thématique, l'ensemble impose, implacable, une cohérence. Déjà, les premier et dernier textes, "Pour le sujet icien d'hui ", "Et ainsi de suis-je», reprennent tous deux le titre du recueil réalisant ainsi un premier bouclage, installant un effet de cadre. Ce seul élément paratextuel et textuel comporte la révélation de certains procédés d'écriture mis en oeuvre dans le recueil et affiche la volonté de l'écrivain de s'inscrire en rupture d'une certaine littérature de la tradition. Dans un écrit théorique au sujet de Nelligan ${ }^{1}$, on retrouve, entre parenthèses, ces mots que je cite volontairement hors contexte parce qu'ils ne m'importent qu'en tant que consigne d'oralité: " sinon les dire : oraux, pour sortir $\mathrm{d}^{\prime \prime}$ une nuit" ". Cette nuit, où l'on voudra bien reconnaître celle du titre à élucider, c'est aussi, comme une note l'explicite à la fin du même texte théorique, la "Nuit" du sonnet "Charles Baudelaire * d'Émile Nelligan, celle des "Classiques * s'opposant à l'«Aurore " du moderne, représenté par Charles Baudelaire dont le nom est hypogrammisé au fil même du poème. (On me pardonnera, je l'espère, ce rappel en forme de raccourci qui trop oublie de se conformer aux strictes règles de la citation). On le devine, un programme se dessine, qui nous intéresse. Tout d'abord, pour peu que l'on tente d'articuler les mots de ces textes, on constate qu'un signifiant peut en cacher un autre, voire qu'une langue peut en cacher une autre. Ainsi, déjà, dans l'incipit du texte-seuil, * Pour le sujet icien d'hui, le corps est là ", se devine originelle, la langue latine de cette expression juridique: "habeas corpus ad subjiciendum ». C'est la citation à comparaître. La réponse à cette injonction qui s'adresse ici au sujet écrivant, n'est autre dans le texte qu'un

1 André Gervais, "Le Vaisseau d'Or": texte et après-textes", Proţe, Chicoutimi, vol. 15, $n^{\circ} 1$, Chicoutimi, 1987, p. 31-43. 
déploiement de stratégies hypogrammatiques à partir du nom de l'auteur. Par exemple ces mots, *Avis déranger *, introduits en bonne et due forme de placard au milieu du texte «Verse et Var "; ils ne sont en fait que l'anagramme du nom, André Gervais. D'entrée de jeu, le texte désamorce sa fiction: le titre d'allure thématique s'avère en fait pleinement métatextuel de même que le sujet convoqué, l'auteur, ne s'avère que dans la dissémination de la matière littérale de son nom.

Je veux donner encore quelque exemple de tels procédés et de leurs répercussions sur l'ensemble du recueil. Le titre de cet autre texte disposé au centre du recueil: * And so on, au seuil ». Par jeu d'hypogramme et par autoréférentialité, il nous ramène au seuil du livre, au premier mot de son paratexte:And(ré). En même temps, et cette fois par échange interlinguistique entre l'anglais et le français, s'opère un nouveau bouclage avec le dernier texte "Et ainsi de suis-je" .

$\mathrm{Si}$, comme nous l'avons vu, le titre du recueil fonctionne un peu comme une citation, il ne constitue pas le seul recours citationnel qui puisse être remarqué au fil de ces pages, loin de là. Ici entre en jeu la très vaste érudition de l'auteur en même temps que s'affirment ses convictions personnelles. Il y a d'abord la citation directe ou devrais-je dire, toutes vérifications n'étant pas faites, ce qui en a tout l'air, avec comme mode d'attribution de simples initiales constituant une litote qu'il n'est pas toujours aisé de résoudre. À titre d'exemple, cette citation tirée d'«Entre fils et coins» (p. 13):

La lecture, c'est avant tout l'art difficile de faire la sourde oreille à toutes les avances par lesquelles un texte voudrait vous faire croire qu'il vous parle. [...] Car si on ne commande pas au verbe, il n'est pas moins impossible d'obéir à la lettre.

D.H.

Il y a encore l'emprunt ou l'allusion comme ces propos de Jean-Pierre Vidal que l'on retrouve ici dans "Questions sans personne à l'oreille du jour " "[il est l']étymologiste qui produit ce qu'il remonte en feinte " ${ }^{2}$. Ces mots de Vidal se rapportent à l'écrivain dans "sa liberté baptiste * et en

2 Jean-Pierre Vidal, * Le souverain s'avarie. Lecture de l'onomastique R-G " (1975) dans Jean Ricardou (sous la direction de), Robbe-Grillet: analyse, theorie. Roman/ cinéma, coll. * 10/18\%, n 1079, Paris, UGE, 1976, p. 273-322. 
96

disent long sur le caractère fictionnel du nom. Pour André Gervais, l'auteur est avant tout «prédauteur * et «scrapveneur». Sur son parcours, il accumule les matériaux à partir desquels il s'acquittera de sa tâche scripturale. Ici, c'est bien, à n'en pas douter, le concept même de *création * qui est mis en cause.

Tout comme sa posture théorique, l'écriture d'André Gervais (c'est ici la même chose en fait) est radicale et les exigences qu'elle pose au lecteur ne le sont pas moins. Il allie et noue, à sa manière, et sans concession à quelque fin de lisibilité que ce soit, des procédés tels le "pun " joycien et les inventions oulipiennes. Dans * De trop * (p. 137 et 149):

C'est en ceci, dit-il, qu'on devient citation.

C'est en forgeant, dites-vous qu'on devient suicide-and-seek avec ${ }^{\odot}$ adavrature, fors je rond.

Pour clore ce trop bref parcours, je reviens à la quatrième de couverture où l'on peut lire: "Quelque part sur la leçon d'identité, La nuit se lève." Cette leçon, ce n'est pas ici que celle du nom qui se dit autre en sa matière littérale. Elle est aussi celle qui nous apprend le retour du même, la littérature. Ici, l'acte scriptural crée le heurt et laisse à la pensée du lecteur la mesure de l'écart entre le sujet écrivant et le sujet de l'écriture, entre une langue et une autre, entre le littéraire et la réalité qu'elle ne sait représenter.

Il eût fallu parler encore d'une connaissance exceptionnelle de l'oeuvre de Marcel Duchamp et de la manière toute spécifique qu'elle trouve ici de s'investir, comme dans ce mot, l'incre, à mi-chemin entre le français et l'anglais, et dans ses avatars tels * link * ou * l'inc ".

En regard de l'écriture d'André Gervais, des questions qu'elle soulève, de son importance territoriale, entre l'ici et l'ailleurs, mes propos ne sont malheureusement, je le crains, que fort superficiels. Il faudra lire, absolument, et reconnaître.

Je termine en soulignant la rare qualité de la conception graphique du recueil et la pertinence de ce tableau de Bruno Santerre présenté en couverture. Le tableau est, pour une rare fois, plus qu'une illustration, il instaure véritablement un dialogue avec l'oeuvre écrite. 